\title{
Distribution and Ecological Risk Assessment of Polycyclic Aromatic Hydrocarbons in Agricultural Soil along Main Roadside in Changchun
}

\author{
Yanan Chen, Jiquan Zhang*, Feng Zhang, Mo Zhou
}

School of Environment, Northeast Normal University, Institute of Natural Disaster Research, Northeast Normal University, Changchun 130024, China

\section{长春市公路沿线农田土壤多环芳烃分析及生态 风险评价}

\author{
陈亚南, 张继权*, 张峰, 周沫 \\ 自然灾害研究所, 东北师范大学环境学院, 长春 130024, 中国
}

\begin{abstract}
Based on theanalysis of agriculturalsoil sampled along main roadside in Changchun. Get the pollution of polycyclic aromatic hydrocarbons in soil, distribution feature of polycyclic aromatic hydrocarbons (PAHs) in agricultural along main roadside in Changchun was investigated; Results showed that the total content of polycyclic aromatic hydrocarbons in soil along Highway in Changchun range 1572.4-4390.2 $\mu \mathrm{g}$, at a high level, and 3 4 rings were the main pollutions. Evaluation of the soils with the N.L.Nemerow composite index and the standard for PAHs management of agricultural soils in Canada showed $57 \%$ of the samples reached the level of heavy pollution; $29 \%$ of the samples reached the level of moderate pollution and the level of light pollution. Evaluation of the agricultural soil along main rode in Changchun had been polluted more serious.
\end{abstract}

Keywords: roadside; agricultural soils;

*通讯作者: 张继权, Zhangjq022@nenu.edu.cn polycyclic aromatic hydrocarbons; risk assessment

摘要

通过对长春市 7 条主要公路沿线农田土壤采 样分析, 得出了这些公路沿线农田土壤中多环 芳烃的污染情况, 分析了多环芳烃在土壤中的 含量与分布。结果表明, 长春市公路沿线农田 土壤多环芳烃总含量范围在 $1572.4-4390.2 \mu \mathrm{g} / \mathrm{kg}$, 处于较高水平, 以 $3 \sim 4$ 环芳烃为主。以加拿大农业区域土壤的 PAHs 的治理标准值为指标, 用内梅罗指数法对土壤 中多环芳烃的污染进行了风险评价。结果表明, 研究区农田土壤中, 重度污染地区占到 57\%, 中度污染占 $29 \%$, 轻度污染占 $14 \%$, 说明长春 市公路沿线农田土壤中多环芳烃污染较为严 重。

关键词: 公路; 农田土壤; 多环芳烃; 风险评 价

\section{1. 引言}

多环芳烃(Polycyclic aromatic hydrocarbons, 简 
Risk Analysis and Crisis Response in Big Data Era (RAC-16)

称 PAHs), 从广义上讲是指由两个或两个以上 苯环构成的化合物,根据苯环的连接方式可以 分为联苯类、多苯代脂肪烃和稠环芳香烃. 狭 义上讲是指由多个苯环或是苯环和环戊二烯 稠合在一起组成的稠环芳香烃类。多环芳烃是 环境中广泛存在的一类具有致畸、致癌、致突 变的有机污染物 [1-5], 是最早发现且数量最多 的致癌物,目前已经发现的致癌性多环芳烃及 其衍生物已超过 400 种,每年排放到大气中的 多环芳烃约几十万吨, 除了一些天然来源之外, 它们主要来自于化石燃料如煤、石油和木材的 不完全燃烧[6-10]。1976年美国环保局 (USEPA) 提出的 129 种 “优先污染物” 中, 多环芳烃类 化合物占了 16 种 [11], 其中 7 种被列入 “中国 环境优先控制污染物”黑名单。

随着社会经济的发展, 大量公路被不断开 发, 导致现如今很多农田被公路包围, 土壤是 多环芳烃的储库和中转站。未使用清洁燃料和 净化器的机动车尾气中的致癌多环芳烃含量
很高。这类污染物以气体或飘尘形式排放到大 气中, 经过沉降作用或雨水冲刷,最终进入土壤 [12]。近年来, 已有对生物工厂引起的污染进 行的风险评价[13], 但是有关长春市公路沿线 农田土壤多环芳烃分析及生态风险评价还没 有相关的研究, 本研究以长春市公路沿线农田 土壤为研究对象, 探究其多环芳烃的含量特征 并进行初步的生态风险评价。

\section{2. 材料与方法}

\section{1. 样品的采集}

依据《土壤环境监测技术规范》(HJ/T166-2004) 的布点和采样的原则, 选择长春市 7 条主要公 路沿线农田采用随机采样法选择 5 点采集耕 层 $(0 \sim 15 \mathrm{~cm})$ 土壤, 将所采土样混匀并装入 聚乙烯塑料袋, 并表明采样编号及地点, 土样 避光风干, 去除植物残根, 过 $2 \mathrm{~mm}$ 篮, 避光 保存。采样位置如图 1。

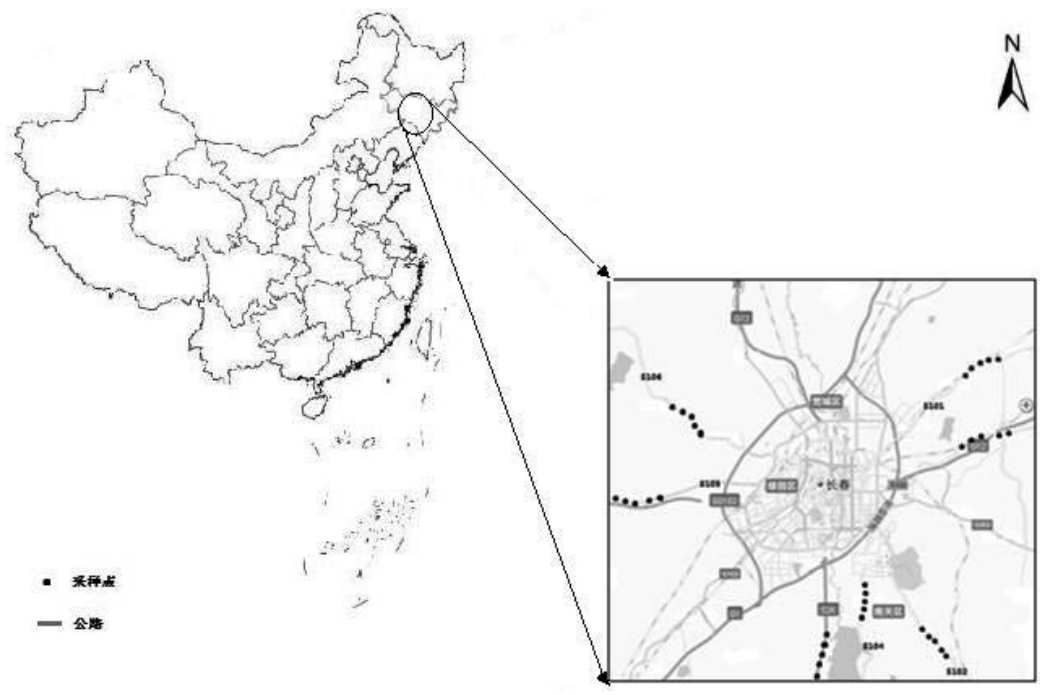

图 1 采样点示意图

\section{2. 分析方法}

称取 5 克土样, 加入 5 克无水硫酸钠和 5 克铜 粉, 以 $40 \mathrm{ml}$ 二氯甲烷: 丙酮 $=1: 1$ 超声萃取 $15 \mathrm{~min}$, 萃取 3 次, 提取液加入到弗罗里硅固 相萃取柱, 并用 $60 \mathrm{ml}$ 的正已烷: 二氯甲烷 $=7: 3$ 进行洗脱, 洗脱液经旋转蒸发仪浓缩至近干,
加入 $10 \mathrm{ml}$ 正己烷, 继续浓缩至 $1 \mathrm{ml}$ 供色谱分 析。

Clarus 680 GC,FID 检测器, HP-5 色谱柱, 载气为氦气, 进样速度为 $1 \mathrm{~mL} / \mathrm{min}$ 。不分流进 样。程序升温: $50^{\circ} \mathrm{C}$ (保持 $2 \mathrm{~min}$ ) 以 $19^{\circ} \mathrm{C} / \mathrm{min}$ 的速度升到 $200^{\circ} \mathrm{C}$ (保持 $2 \mathrm{~min}$ ), 以 $4.5^{\circ} \mathrm{C} / \mathrm{min}$ 的速度升到 $240^{\circ} \mathrm{C}$ (保持 $2 \mathrm{~min}$ ), 以 $2.5^{\circ} \mathrm{C} / \mathrm{min}$ 
Risk Analysis and Crisis Response in Big Data Era (RAC-16)

的速度升到 $300^{\circ} \mathrm{C}$ (保持 $5 \mathrm{~min}$ )。标准样品为 美国环保局（USEPA）颁布的 16 种多环芳烃 混标, 分别为: naphthalene (Nap) 荎, acenaphthene (Ace)范, acenaphthylene (Acy)范 烯, fluorene (Flo) 芴, phenathrene (Phe) 菲, anthracene (Ant) 蒽, fluoranthene (Fla) 苂萝, pyrene (Pyr)萠, benzo(a)anthracene $(\mathrm{BaA})$ 苯并 (a)蒽, chrysene (Chr)䓛, benzo(b)fluoranthene $(\mathrm{BbF})$ 苯并 $(\mathrm{b})$ 苂葱, benzo(k)fluoranthene $(\mathrm{BkF})$ 苯并 $(k)$ 荧蒽, benzo(a)pyrene $(\mathrm{BaP})$ 苯并 $(\mathrm{a})$ 萠, $\operatorname{dibenz}(\mathrm{a}, \mathrm{h})$ anthracene $(\mathrm{DahA})$ 二苯并 $(\mathrm{a}, \mathrm{h})$ 葱, indeno(1,2,3-cd)pyrene (IcdP) 茆苯 $(1,2,3-c d)$ 芘 and benzo(g,h,i)perylene (BghiP) 苯并 $(\mathrm{g}, \mathrm{h}, \mathrm{i})$ 菲。

\section{1. 多环芳烃在土壤中的含量与分布}

监测结果表明,长春市公路沿线农田土壤中 16 种优控 PAH 总残留量检测范围在 1572.4-4390.2ng/g 之间。检出率较低的是狮苯 (1,2,3-cd) 萠和苯并 $(\mathrm{g}, \mathrm{h}, \mathrm{i})$ 菲, 检出率均为 $42.8 \%$, 其余组分检出率皆为 $100 \%$ 。PAHs 检出率如此 之高, 说明长春地区公路沿线农田土壤 PAHs 污染普遍。单一污染物以荎、范烯、菲、葱、 萠、苯并 $(a)$ 葱、䓛、苯并 $(b)$ 苂葱、苯并 $(k)$ 苂 葱、二苯并 $(a, h)$ 蒽和狮苯 $(1,2,3-c d)$ 萠为主。表 1 为长春公路沿线农田土壤多环芳烃含量。各 单体多环芳烃变异系数较大, 表明该地区多环 芳烃含量受人为影响较大。

\section{3. 结果与讨论}

表 1 长春公路沿线农田土壤多环芳烃含量

\begin{tabular}{|c|c|c|c|}
\hline PAHs $(\mu \mathrm{g} / \mathrm{kg})$ & 含量范围 & Mean & C. $v \% \mathrm{a}$ \\
\hline 蒜 Nap & $243.1-673.8$ & 449.1 & 38 \\
\hline 范 Ace & $14.1-38.3$ & 25.2 & 38 \\
\hline 范烯 Acy & $135.7-672.2$ & 465 & 41 \\
\hline 芴 Flo & $18.9-34$ & 24.3 & 23 \\
\hline 菲 Phe & $179.6-372.9$ & 249 & 30 \\
\hline 蒽 Ant & $196.7-588.4$ & 362.4 & 44 \\
\hline 苂蒽 Fla & $30.1-95.7$ & 66.3 & 36 \\
\hline 芘 Pyr & $72.1-498.7$ & 244.5 & 68 \\
\hline 苯并(a)蒽 $\mathrm{BaA}$ & $48.6-354.4$ & 179.4 & 75 \\
\hline 䓛Chr & $116.7-393.3$ & 205.3 & 51 \\
\hline 苯并(b)苂蒽 BbF & $51.2-273.2$ & 137.8 & 72 \\
\hline 苯并 $(\mathrm{k})$ 菼蒽 BkF & 70.9-339.1 & 175.1 & 61 \\
\hline 苯并(a)芘 $\mathrm{BaP}$ & $49.2-118.7$ & 83.6 & 32 \\
\hline 二苯并 $(\mathrm{a}, \mathrm{h})$ 蒽 $\mathrm{DahA}$ & $50.7-293.7$ & 177 & 51 \\
\hline 狮苯 $(1,2,3-c d)$ 芘 IcdP & ND-247.8 & 190.6 & 38 \\
\hline 苯并(g,h,i)菲 BghiP & ND-93.7 & 68.5 & 44 \\
\hline 16 PAHs & $1572.4-4390.2$ & 2954.9 & 33 \\
\hline
\end{tabular}

a 变异系数; ND: 未检出.

\section{2. 不同环数多环芳烃分布情况}

研究表明, 2 环、3 环等低环 PAHs 一般表现 出较强的急性毒性, 而高环数的 PAHs 却对许 多生物有 “三致” 作用[14-16]。PAHs 环数相 对丰度可以反映来自热解或石油类污染, 通常 4 环及 4 环以上 PAHs 主要来源于化石燃料高 温燃烧, 而 2 环和 3 环 PAHs 则来源于石油类污
染[17-19]。表 2 为各采样点各多环芳烃含量百 分比, 从表中可以看出, 公路沿线农田土壤中 多环芳烃主要以 3 环和 4 环为主, 说明该研究 区多环芳烃主要来自于石油类污染及化石燃 料高温燃烧, 该结果正好印证了公路汽车尾气 的排放及油沥青等可能为多环芳烃的主要污 染来源。 
Risk Analysis and Crisis Response in Big Data Era (RAC-16)

表 2 各采样点各环数多环芳烃含量 (\%)

\begin{tabular}{lccccc}
\hline 采样点 & $\mathbf{2}$ 环 & $\mathbf{3}$ 环 & 4 环 & $\mathbf{5}$ 环 & $\mathbf{6}$ 环 \\
\hline S102 & 15.46 & 34.66 & 31.77 & 15.87 & 2.25 \\
S104 & 12.90 & 44.44 & 25.14 & 17.54 & 0 \\
S26 & 15.94 & 29.91 & 24.41 & 24.13 & 5.61 \\
S105 & 14.01 & 52.45 & 18.41 & 11.59 & 3.56 \\
S106 & 15.35 & 28.19 & 25.35 & 23.35 & 7.78 \\
G12 & 13.71 & 42.75 & 18.08 & 21.84 & 3.63 \\
S101 & 17.94 & 44.15 & 23.31 & 14.61 & 0 \\
平均值 & 15.05 & 39.51 & 23.78 & 18.41 & 3.26 \\
\hline
\end{tabular}

\section{3. 土壤中多环芳烃污染评价}

由于我国现行的 GB15618-1995《土壤环境质 量标准》中没有涉及到有关于农田土壤多环芳 烃的标准, 故本研究采用加拿大农业土壤多环 芳烃的治理标准值。该标准规定, 农业土壤中 萗、菲、芘、苯并(a)葱、苯并 $(a)$ 芘、苯并 $(b)$ 苂葱、苯并 $(k)$ 苂葱、二苯并 $(a, h)$ 葱和狮苯 (1,2,3-cd) 萠的环境健康控制标准为 $100 \mathrm{ng} / \mathrm{g}[20]$ 。

本研究对 7 条公路沿线农田土壤中的上 述 9 种多环芳烃含量采用内梅罗综合污染指 数 $\left(\mathrm{P}_{\mathrm{N}}\right)$ 进行生态风险评价。计算公式如下:

$$
\begin{aligned}
\bar{P}_{i} & =\frac{1}{n} \sum_{i=1}^{n} P_{i} \\
P_{N} & =\sqrt{\frac{\bar{P}_{i}^{2}+P_{i \max }^{2}}{2}}
\end{aligned}
$$

式中 $\bar{P}_{i}$ 为土壤中单项污染指数平均值; $P_{i \max }$ 为土壤中单向污染指数 $P_{i}$ 中的最大值; $\mathrm{n}$ 为单向污染指数个数。按照 $P_{N}$ 值, 可以将土 壤分为安全 $\left(P_{N} \leq 0.7\right)$ 、警戒线 $\left(0.7<P_{N} \leq 1.0\right)$ 、 轻污染 $\left(1.0<P_{N} \leq 2.0\right)$ 、中污染 $\left(2.0<P_{N} \leq 3.0\right)$ 和重污染 $\left(P_{N} \geq 3.0\right) 5$ 个安全级别。计算得到 的 $P_{N}$ 值和安全级别列于表 3 。 表 3 各采样点内梅罗综合污染指数 (PN) 和安全级别

\begin{tabular}{ccc}
\hline 采样点 & $\boldsymbol{P}_{\boldsymbol{N}}$ & 安全级别 \\
\hline $\mathrm{S} 104$ & 1.84 & 轻污染 \\
$\mathrm{S} 26$ & 2.42 & 中污染 \\
$\mathrm{S} 105$ & 4.71 & 重污染 \\
$\mathrm{S} 106$ & 2.25 & 中污染 \\
$\mathrm{G} 12$ & 5.27 & 重污染 \\
$\mathrm{S} 101$ & 3.25 & 重污染 \\
$\mathrm{S} 104$ & 4.34 & 重污染 \\
\hline
\end{tabular}

由表 3 可以看出, $P_{N}$ 值的范围在 1.84-5.27 之间, 全部采样点中, 重度污染地区占到 57\%, 中度污染占 $29 \%$, 轻度污染占 $14 \%$ 。表明公路 沿线农田土壤收到多环芳烃的污染是值得注 意的。

\section{4. 结论}

土壤样品中 PAHs 总量为 $164.16 \sim 341.46 \mu \mathrm{g} / \mathrm{kg}$, 低于美国 E P A 的环境标准,但是其含量仍然 高于一些环境背景值。研究区土壤中多环芳烃
结构主要以 3 4 环为主, 主要来自于石油类污 染及化石燃料的高温燃烧, 汽车尾气的排放可 能是研究区土壤中 PAHs 的重要来源。研究区 生态风险较大, 重度污染地区占到 $57 \%$, 应引 起高度重视, 并采取有力措施进行防治。土壤 中多环芳烃含量高, 不仅直接危害人体健康, 还 能间接污染地下水.土壤中高含量的多环芳烃 还会被植物吸收, 通过食物链中的物质循环累 积在其他生物体内,危害其他动植物的健康. 因 此,农田土壤中 PAHs 含量应该引起重视. 


\section{Acknowledgements}

This study was supported by the National Major Program of Water Pollution Control and Treatment Technology of China under Grant No. 2014ZX07201-011-002) and the National Non-Profit Research Program of China (No.201401015).

\section{致谢}

本文以国家重大水专项（2014ZX07201-011）

和水利部公益性行业科研专项经费项目

（201401015）为项目支撑。

\section{参考文献}

[1] Zhang D, Liu J, Jiang X, Cao K, Yin P, Zhang $\mathrm{X}$. Distribution, sources and ecological risk assessment of PAHs in surface sediments from the Luan River Estuary, China.Marine Pollution Bulletin. 102(1): 223-229, 2016.

[2] Liu F, Liu J, Chen Q, Wang B, Cao Z. Pollution characteristics and ecological risk of polycyclic aromatic hydrocarbons (PAHs) in surface sediments of the southern part of the Haihe River system in China. Chinese Science Bulletin. 58(27): 3348-3356, 2013.

[3] Suman S, Sinha A, Tarafdar A. Polycyclic aromatic hydrocarbons (PAHs) concentration levels, pattern, source identification and soil toxicity assessment in urban traffic soil of Dhanbad, India. Science of The Total Environment. 545-546: 353-360, 2016.

[4] Thea A E, Ferreira D, Brumovsky L A, Schmalko M E. Polycyclic aromatic hydrocarbons (PAHs) in yerba maté (Ilex paraguariensis St. Hil) traditional infusions (mate and tereré).Food Control. 60: 215-220, 2016.

[5] 冯焕银, 傅晓钦, 赵倩, 高占国. 宁波土壤 中多环芳烃的健康风险评价. 农业环境科 学学报. 30(10): 1998-2004, 2011.

[6] Zhu Y, Yang L, Yuan Q, Yan C, Dong C,
Meng C, Sui X, Yao L, Yang F, Lu Y, Wang W. Airborne particulate polycyclic aromatic hydrocarbon (PAH) pollution in a background site in the North China Plain: Concentration, size distribution, toxicity and sources. Science of The Total Environment. 466-467: 357-368, 2014.

[7] Xu J, Guo J, Liu G, Shi G, Guo C, Zhang Yuan, Feng Y. Historical trends of concentrations, source contributions and toxicities for PAHs in dated sediment cores from five lakes in western China.Science of The Total Environment. 470-471: 519-526, 2014.

[8] Qiao M, Wang C, Huang S, Wang D, Wang Z. Composition, sources, and potential toxicological significance of PAHs in the surface sediments of the Meiliang Bay, Taihu Lake, China. Environment International. 32(1): 28-33, 2006.

[9] Liu Y, Wang S, Lohmann R, Yu N, Zhang C, Gao Y, Zhao J, Ma L. Source apportionment of gaseous and particulate PAHs from traffic emission using tunnel measurements in Shanghai, China. Atmospheric Environment. 107: 129-136, 2015.

[10] Chen F, Hu W, Zhong Q. Emissions of particle-phase polycyclic aromatic hydrocarbons (PAHs) in the Fu Gui-shan Tunnel of Nanjing, China. Atmospheric Research. 124: 53-60, 2013.

[11] 曹云者, 柳晓娟, 谢云峰, 张大定, 李发 生. 我国主要地区表层土壤中多环芳烃组 成及含量特征分析. 环境科学学报. 32(01): 197-203, 2012.

[12] 吕金刚, 毕春娟, 陈振楼, 周婕成, 韩景 超. 上海市崇明岛农田土壤中多环芳烃分 布和生态风险评价. 环境科学. 33(12): 4270-4275, 2012.

[13]Kadri F. The Assessment of Risk Caused by Fire and Explosion in Chemical Process Industry: A Domino Effect-Based Study. Journal of Risk Analysis and Crisis Response. 3(2): 66-76, 2013. 
Risk Analysis and Crisis Response in Big Data Era (RAC-16)

[14] 朱媛媛, 田靖, 魏恩琪, 魏复盛. 天津市 土壤多环芳烃污染特征、源解析和生态风 险评价.环境化学. 33(02): 248-255, 2014.

[15] 郑太辉, 再勇, 陈来国. 东江流域农村土 壤中多环芳烃的分布特征及其健康风险评 估. 生态环境学报. 23(04): 657-661, 2014.

[16] 吴维兴. 土壤中多环芳烃污染及其环境 行为研究进展. 安徽农业科学. 42(25): 8563-8565, 2014.

[17] 于波，孙晓怡，唐伟，金永民. 抚顺地区 土壤中多环芳烃分布及污染风险评价. 环
境保护与循环经济. (02): 43-45, 2008.

[18] 聂麦茜, 张志杰. 环境中多环芳烃污染规 律及其生物净化技术. 环境导报. (01): 18-21, 2001.

[19] 朱先磊, 王玉秋, 刘维立, 朱坦. 焦化厂 多环芳烃成分谱特征的研究. 中国环境科 学. 21(03): 75-78, 2001.

[20] 潘峰, 耿秋娟, 楚红杰, 王利利. 石油污 染土壤中多环芳烃分析及生态风险评价. 生态与农村环境学报. 27(05): 42-47, 2011. 